$1 \leqslant r$

دراسة تحليلية لأثر السياسات الزراعية والسعرية على إنتاج واستهلاك بعض المحاصيل الزيتية فى مصر

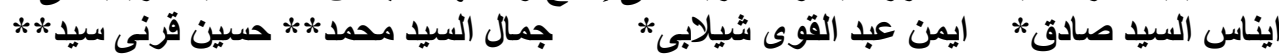

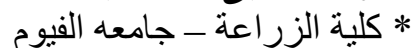

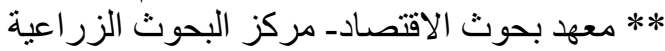

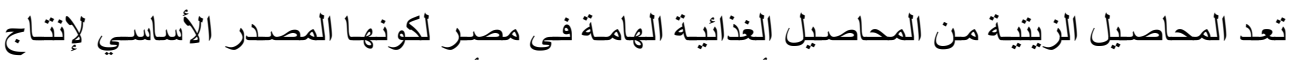

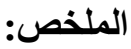

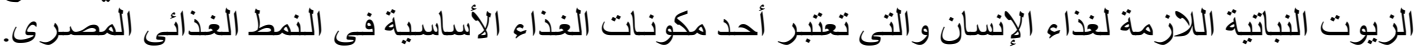

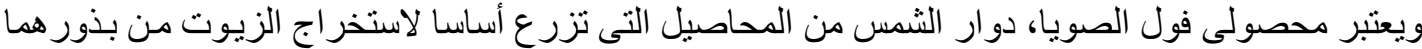

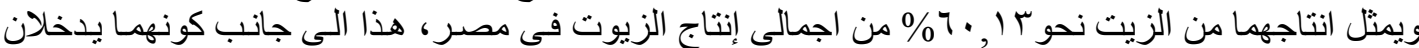

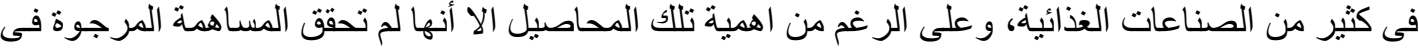

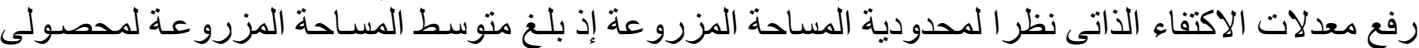

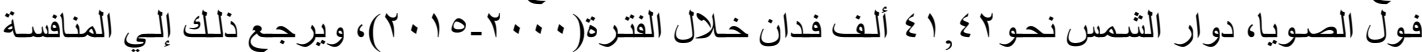

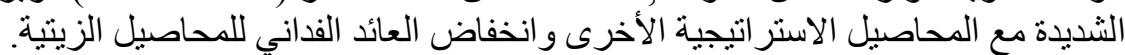

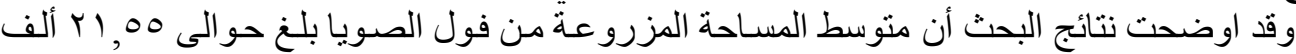

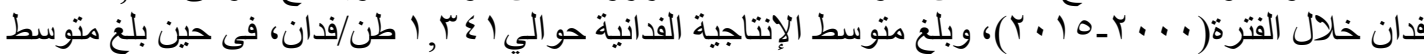

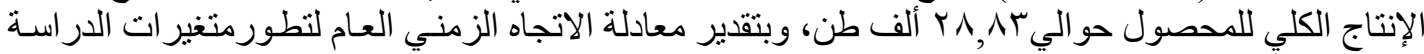

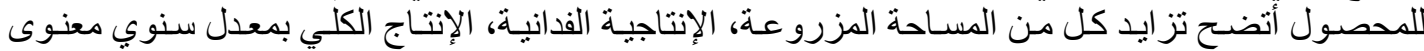

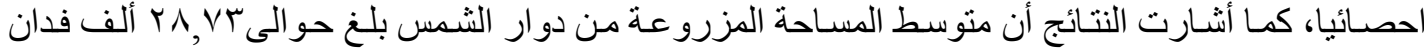

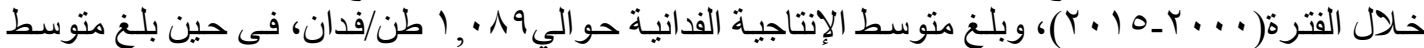

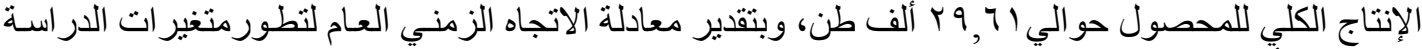

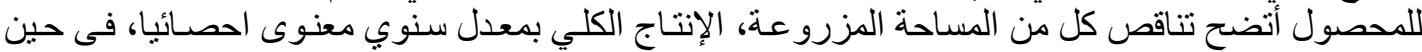

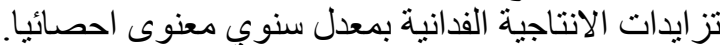

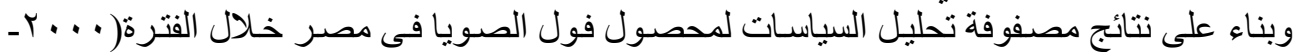

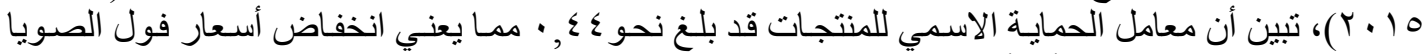

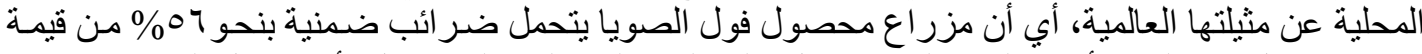

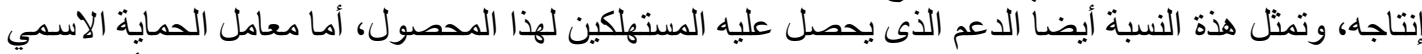

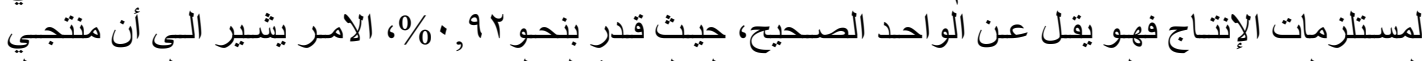

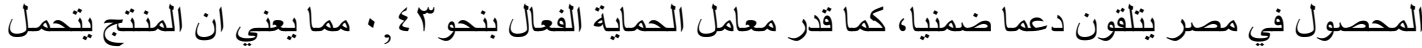

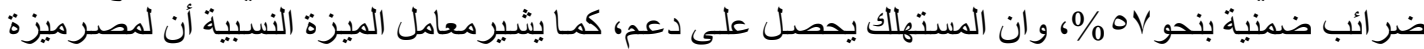

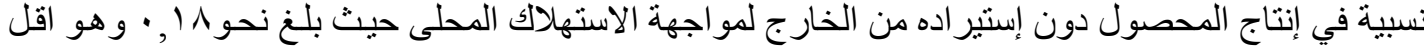

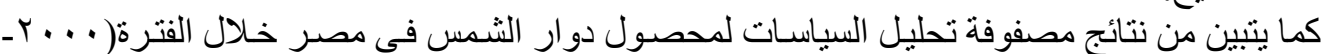

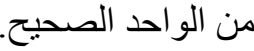

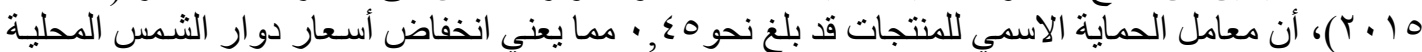

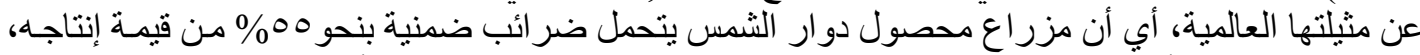

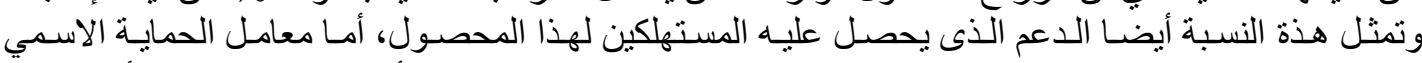

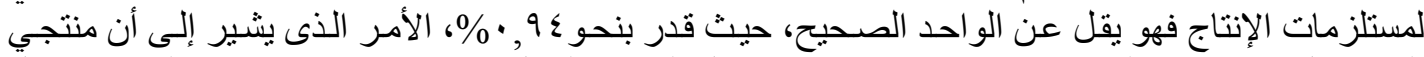

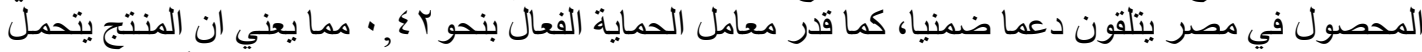

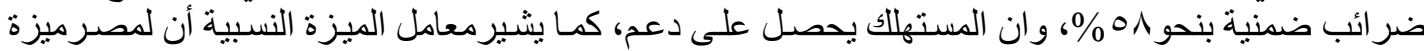

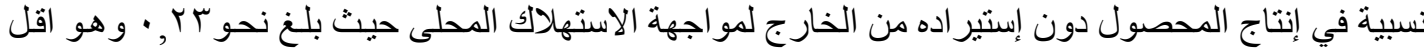

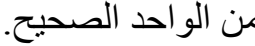
الكلمات الدالة: معاملات الحماية الاسميةــ معامل الحماية الفعالـ معامل الميزة النسبية. مقدمة:

تعتبر محاصيل الزيوت الغذائية ومنتجاتها من الزيوت من السـلع الاستر اتيجية الهامـة فى الاقتصـاد

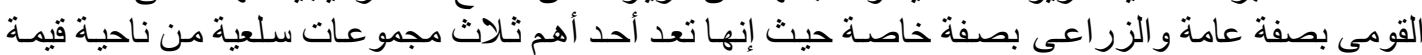

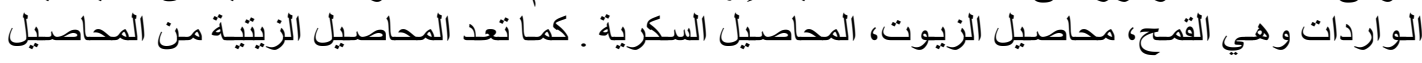

Fayoum J. Agric. Res. \& Dev., Vol. 33, No.1, January, 2019 
$1 \leqslant \mu$

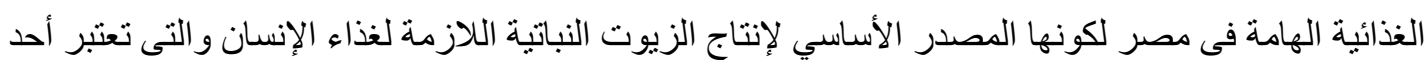

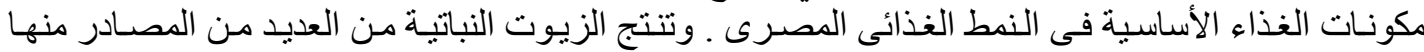

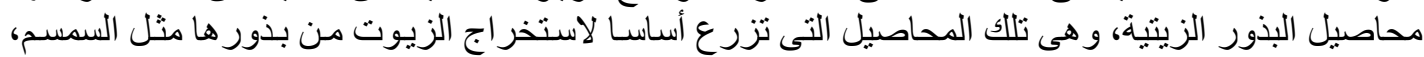

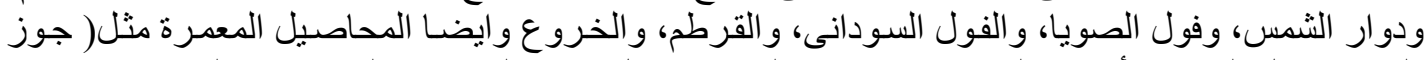

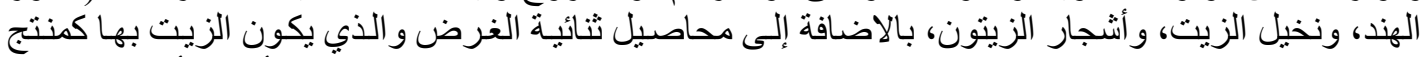

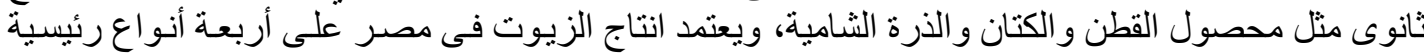

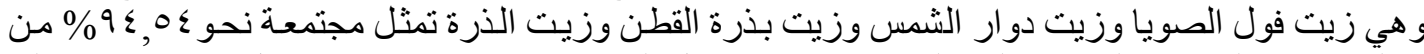

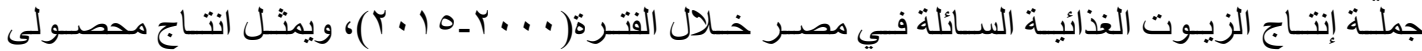

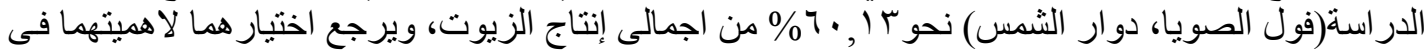

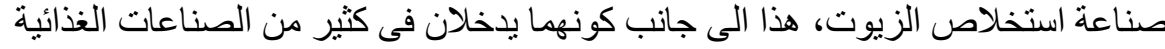

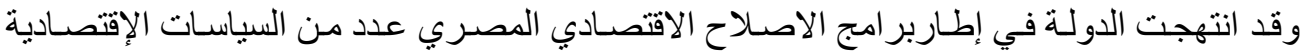

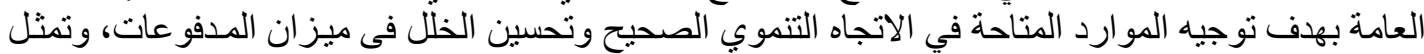

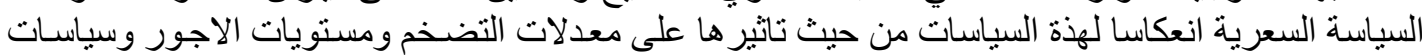

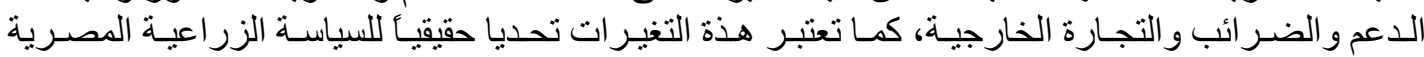

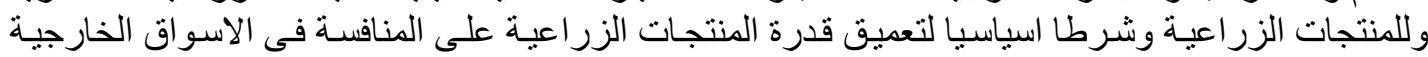

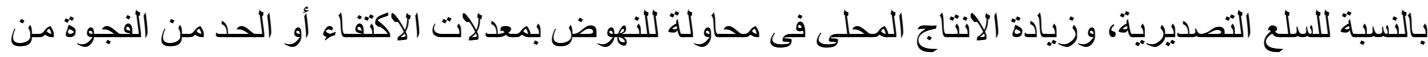

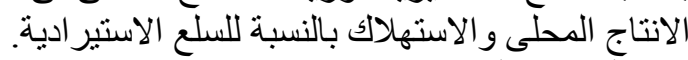

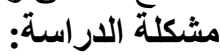

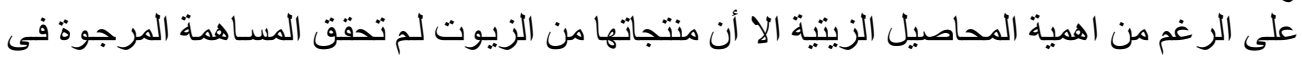

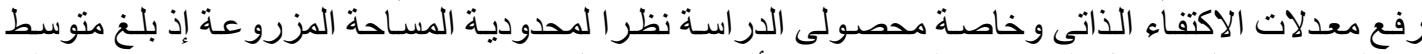

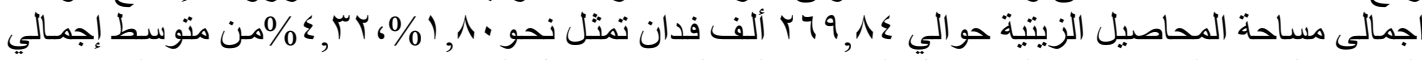

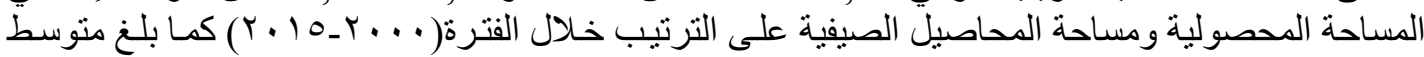

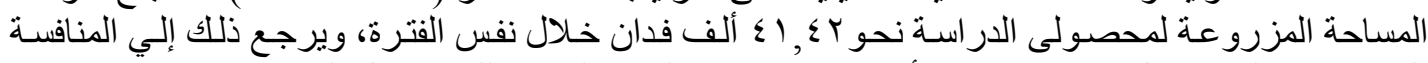

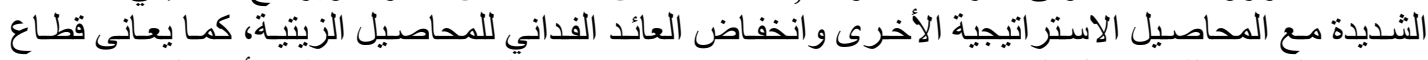

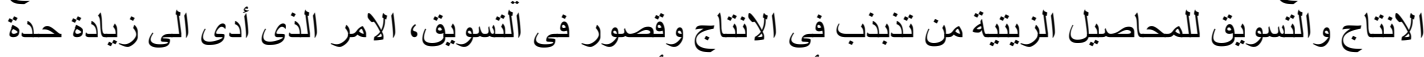

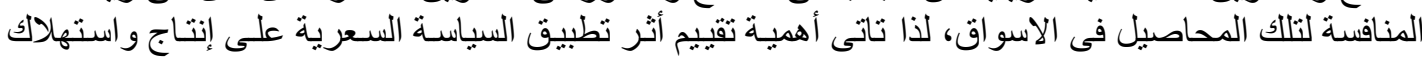

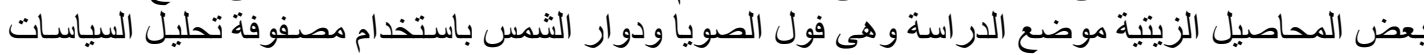

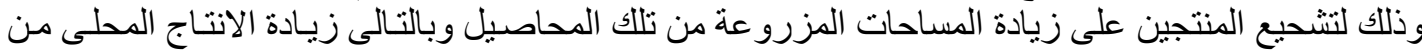
الزيوت و الحد من الفجوة الغذائية للزيوت النئ

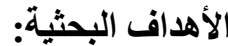

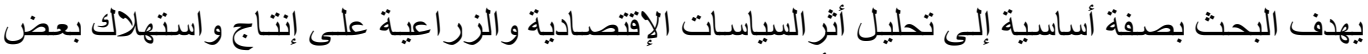
المحاصيل الزيتية في مصر وذللك من خلال الأهداف الفر التية النية التالية:

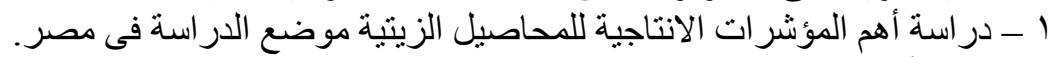

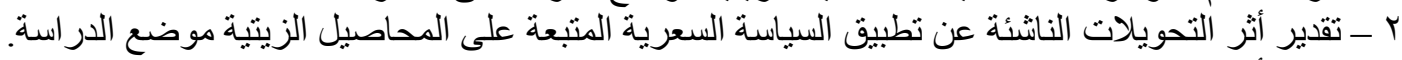

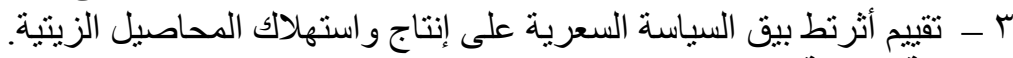
الطريقة البحثية ومصادر البيانات:

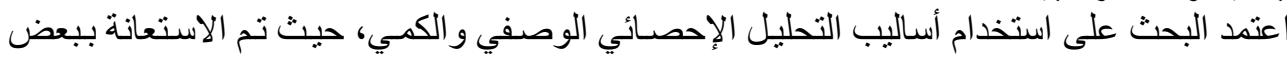

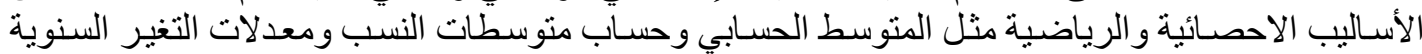

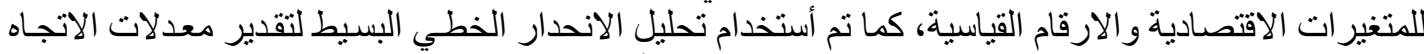

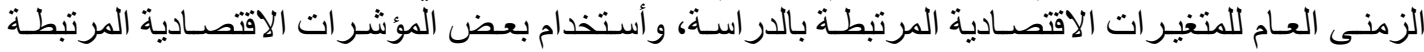

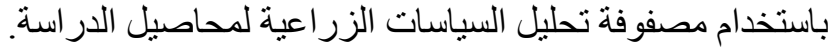

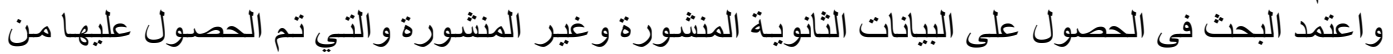

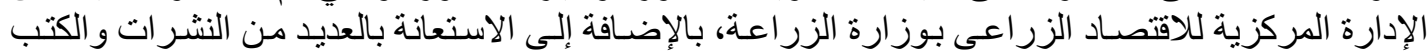

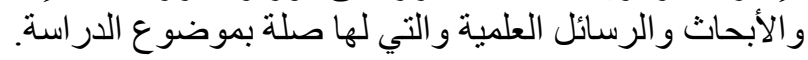

Fayoum J. Agric. Res. \& Dev., Vol. 33, No.1, January, 2019 


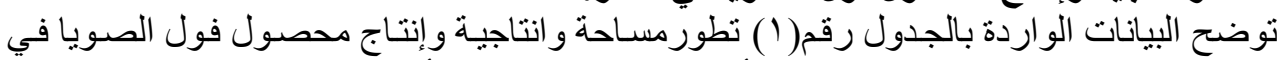

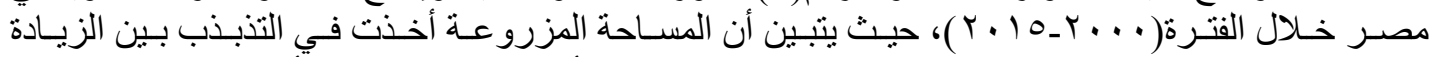

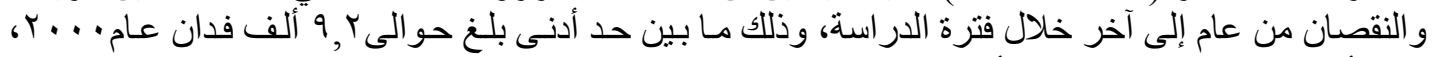

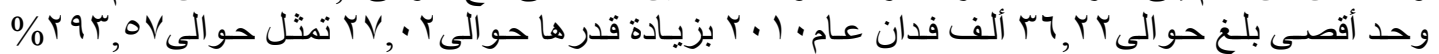

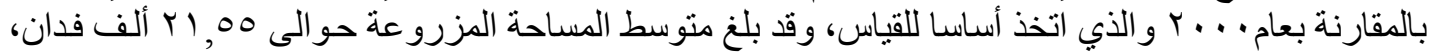

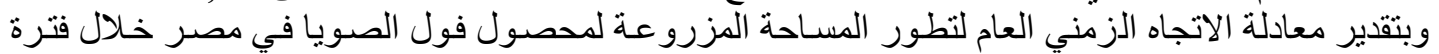

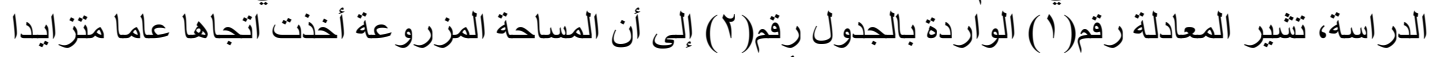

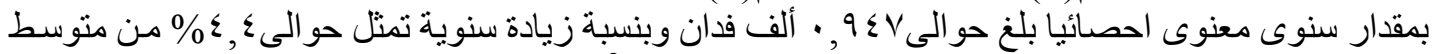

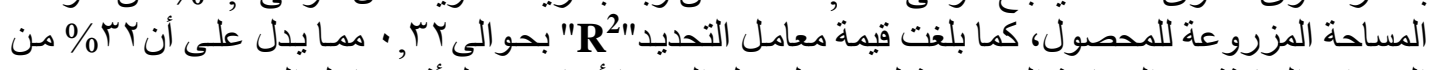

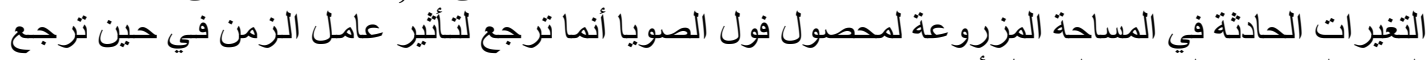

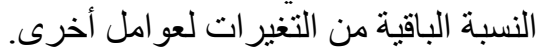

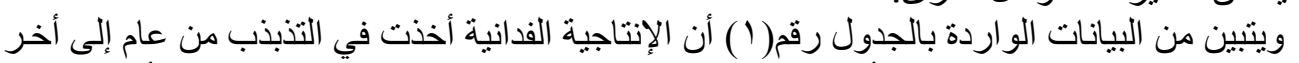

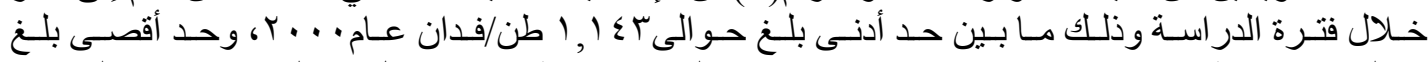

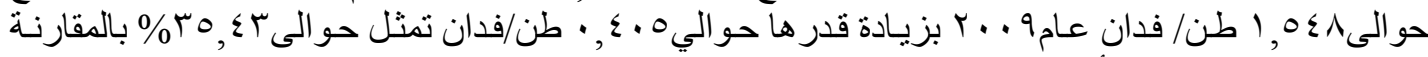

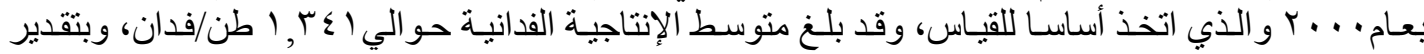

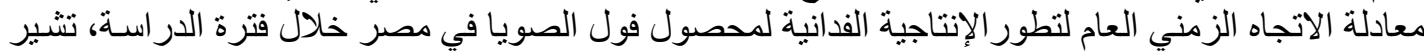

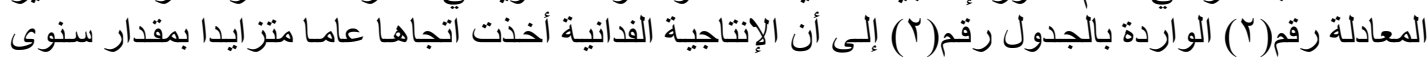

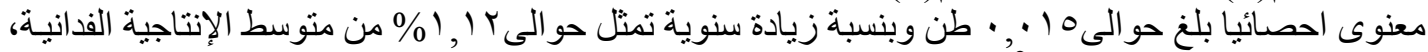

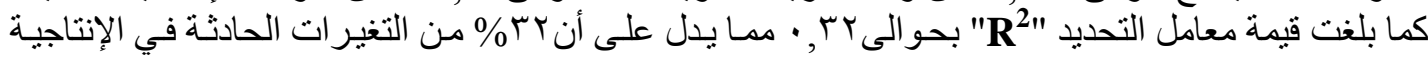

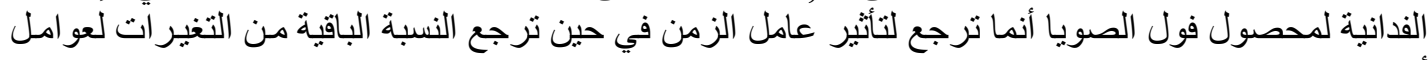
أخرى.

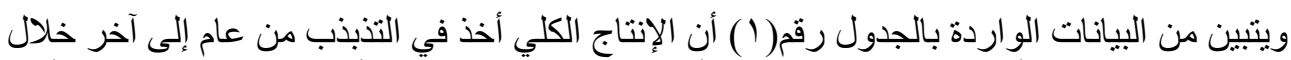

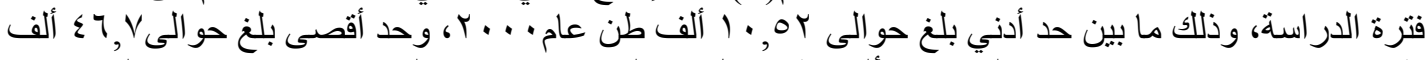

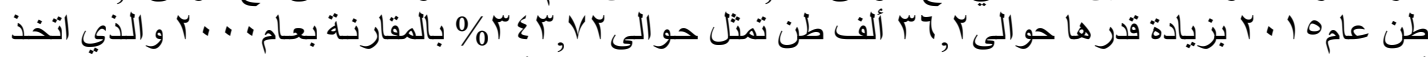

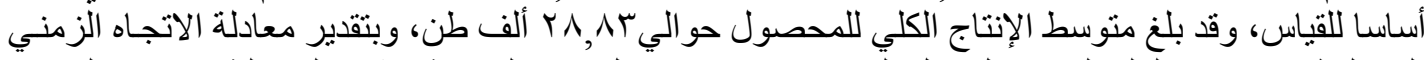

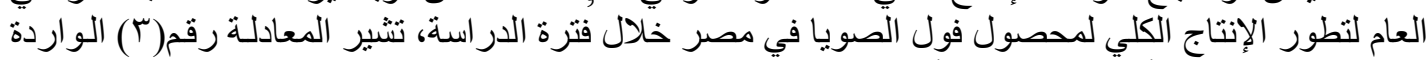

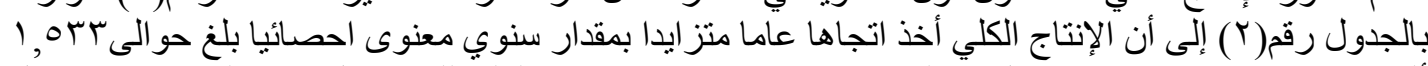

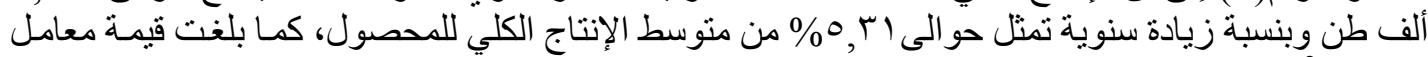

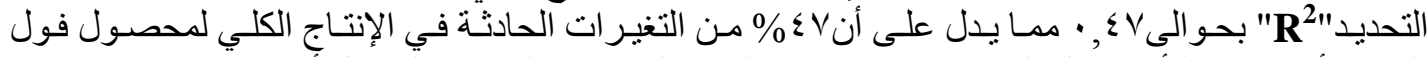

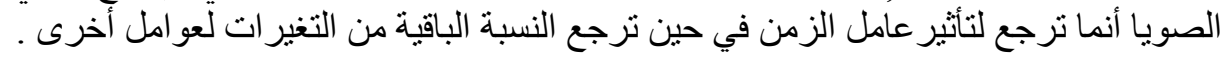

Fayoum J. Agric. Res. \& Dev., Vol. 33, No.1, January, 2019 
جدول رقم ( ) تطور المساحة المزروعة والإنتاجية الفدانية والإنتاج الكلي لمحصول فول الصويا في مصر

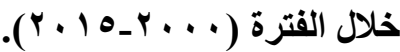

\begin{tabular}{|c|c|c|c|c|c|c|}
\hline 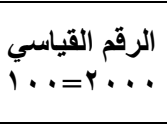 & (بالألفت طن الكلي & الرقم القياسي . . . . . 1 & |الإنتاجية الفدانية & . . . . . الرقم القياسي . & 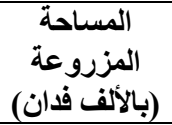 & السنوات \\
\hline $1 \ldots$ & $1 ., 0 Y$ & $1 \ldots$ & $1,1 \leqslant T$ & $1 \ldots$ & $q, Y$. & $r \ldots$ \\
\hline$T r \cdot, T r$ & $1 r, 71$ & I.r,Tr & $1, I V r$ & $\mid r v, \wedge \varepsilon$ & Ir, & $r \ldots l$ \\
\hline 171,19 & $I V, V$ & $1.9, \wedge 1$ & $1, Y 07$ & lor,9V & $1 \leqslant 1$ & $r \ldots r$ \\
\hline TVY,TA & $r \wedge, V$ & ITV,IY & $1, \leqslant 0 r$ & $Y I \leqslant, \leqslant Y$ & $19, v \leqslant$ & $r \ldots r$ \\
\hline 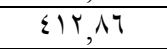 & $\varepsilon r, \varepsilon r$ & $111, \mathrm{rA}$ & I, YVY & $r V I, \cdot r$ & $r \leqslant, 10$ & Y.. \\
\hline$r \leqslant 0, \leqslant 9$ & YO,AY & $11 r, 01$ & $1, Y \wedge T$ & $r \backslash \wedge, I r$ & $r \cdot, 1$ & Y... \\
\hline$r \backslash \wedge, \wedge r$ & $r r, \cdot r$ & $11 r, r 1$ & $1,19 \varepsilon$ & $19 r, r r$ & $I V, \Lambda$ & $Y \ldots Y$ \\
\hline$r \leqslant r, \leqslant 0$ & YO, TI & $1 r \cdot, 9$ & $1, r \wedge r$ & $r \cdot 1, r v$ & 11,04 & $r \ldots V$ \\
\hline TVV,TY & $r q, r$ & $M T, \leqslant \varepsilon$ & $1, \leqslant 11$ & $Y Y \leqslant, 0 T$ & $r \cdot, T V$ & $Y \ldots \lambda$ \\
\hline ro. 91 & $r \bar{r}, \varepsilon$ & $1 \mu_{0}, \leqslant r$ & $1,0 \leqslant \lambda$ & $110, r_{9}$ & $I V, \cdot 0$ & Y.. q \\
\hline$\$ 11,0 \mathrm{~V}$ & $\varepsilon r, r$ & $1 \cdot \leqslant, 0 \leqslant$ & 1,190 & rqr,ov & $r \overline{Y Y Y}$ & $r .1$. \\
\hline rAY,qY & $r q, \Lambda$ & $11 \leqslant, 71$ & $1, \mu 1$. & $r \leqslant T, \lambda r$ & $Y Y, V Y$ & $r .11$ \\
\hline$r \leq 7,7)$ & ro, q $\varepsilon$ & זי, & 1,017 & 110,10 & $1 V, 11$ & $Y . I Y$ \\
\hline$r \|, r \varepsilon$ & rY, Vo & $I T V, V T$ & $1, \leqslant 7$. & $r \leqslant r, T r$ & $Y Y, \leqslant Y$ & $r .1 T$ \\
\hline$r \vee \wedge, 90$ & $r q, \wedge \uparrow$ & IrY,rq & $1, r 99$ & $r \cdot q, \varepsilon \wedge$ & $r \wedge, 0$ & Y.I \\
\hline$\varepsilon \varepsilon r, V Y$ & $\varepsilon\rceil, V$ & $K \cdot, \leqslant V$ & $1, \Gamma V V$ & $r \Psi \Lambda, Y V$ & $r r, q$ & $Y .10$ \\
\hline$T V \varepsilon, \cdot 9$ & $r \wedge, \wedge r$ & $\| V, \leqslant Y$ & $1, \Gamma \leqslant 1$ & $T r \leqslant 10$ & $Y 1,00$ & المتوسط \\
\hline
\end{tabular}

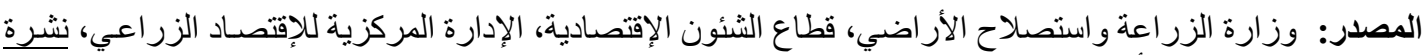
الإحصاءات الزر اعية، أعداد مختلفة.

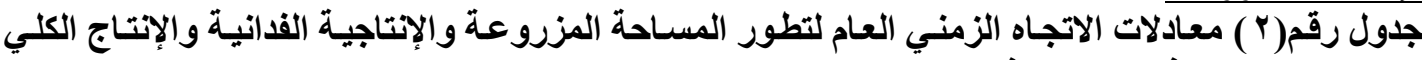

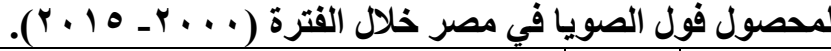

\begin{tabular}{|c|c|c|c|c|c|c|}
\hline $\begin{array}{l}\text { معدل التغيرى \% } \\
\text { \% }\end{array}$ & $\mathbf{F}$ & $\mathbf{R}^{2}$ & & معادلة الاتجاه الزمني العا & المعادلة & المتغير \\
\hline$\xi, \xi$ & $7, \wedge 1$ & $\cdot, r$ & $*(Y, T)$ & ص^ هـ= •, 0 & 1 & المساحة المزروعة \\
\hline 1,14 & $7, \wedge 1$ & $\cdot, r Y$ & $*(Y, T)$ & ص^هـ= | صا & $r$ & الإنتاجية الفدانية \\
\hline 0,4 & Ir,AV & $\cdot, \leqslant V$ & $* *(\Upsilon\urcorner)$, & ص^هـ = 10,11 + 1,0rr س هـ & $r$ & الإنتاج الكلي \\
\hline
\end{tabular}

ص^ هـ = القيمة التقديرية للمتغير ات التابعة (المساحة و الإنتاجية و الإنتاج) في السنة هـ.

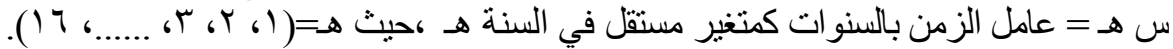

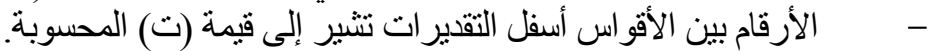

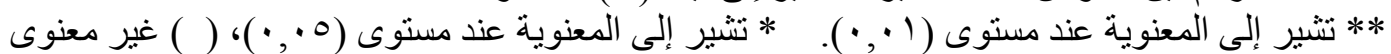

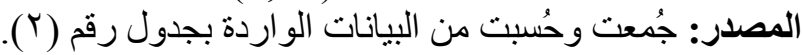

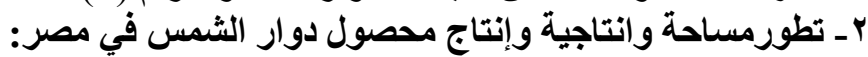

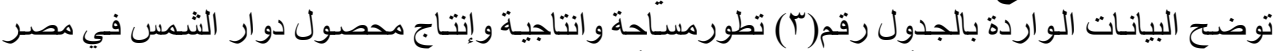

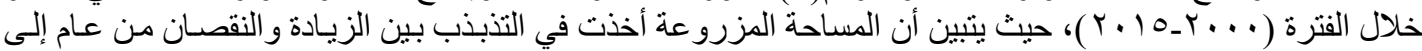

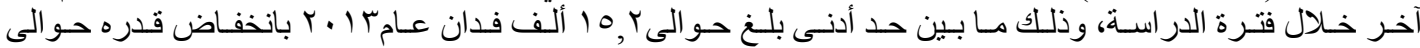

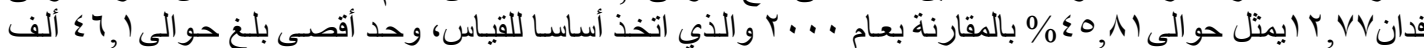

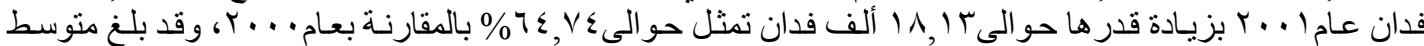

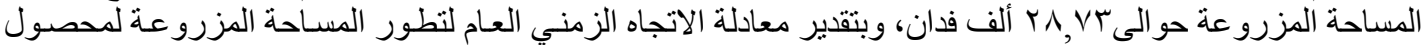

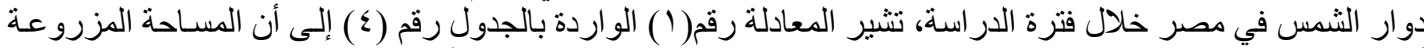

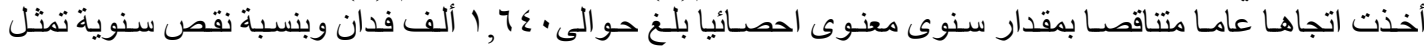

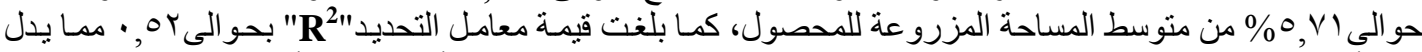

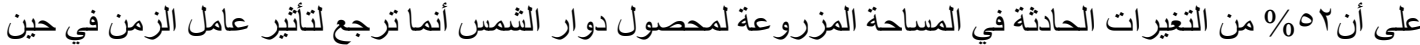
ترجع النسبة الباقية من التغير ات لعوات امل أخرى.

Fayoum J. Agric. Res. \& Dev., Vol. 33, No.1, January, 2019 
$1 \leq 7$

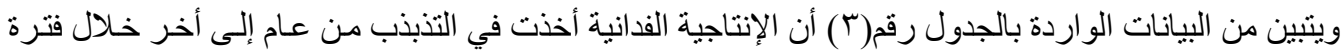

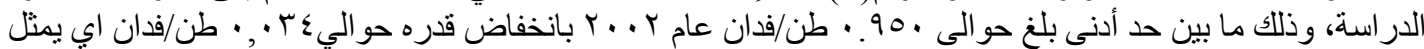

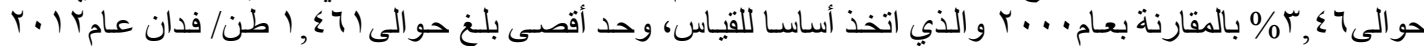

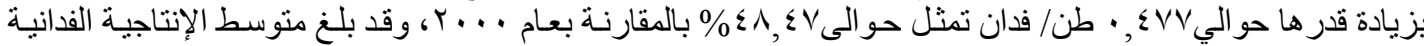

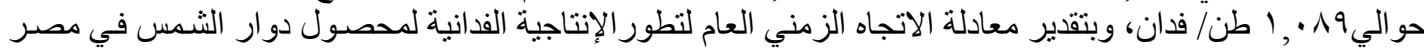

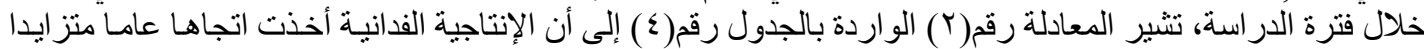

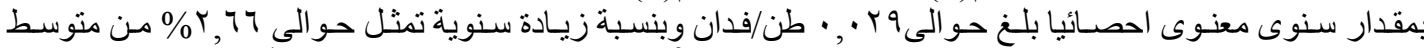

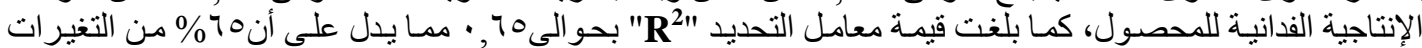

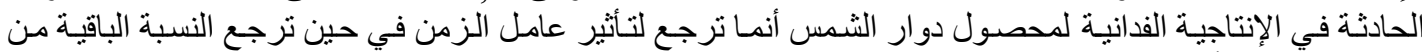

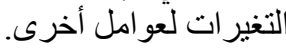

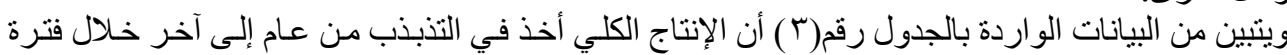

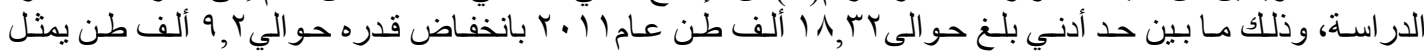

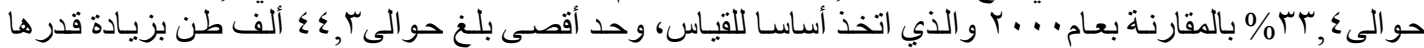

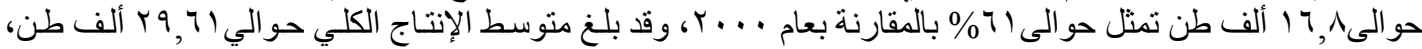

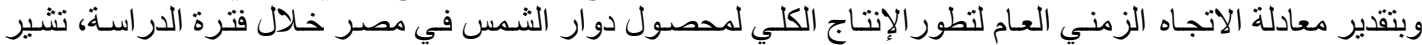

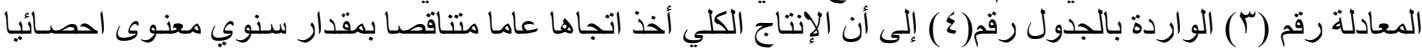

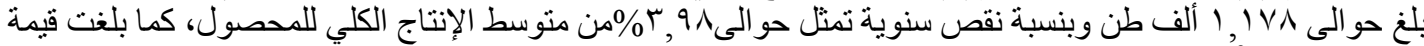

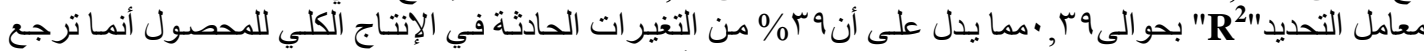

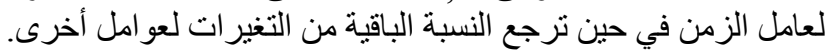

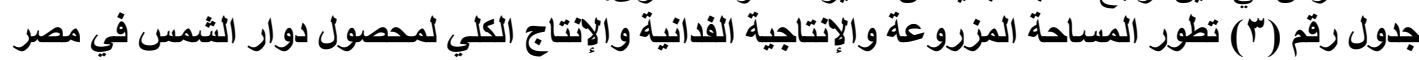

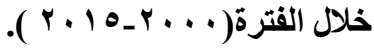

\begin{tabular}{|c|c|c|c|c|c|c|}
\hline الرقم القياسي . . . . . . . & 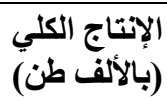 & الرقم القياسي & الإنتاجية القدانية & الرقم القياسي . . . . . . & المساحة المزروعة & السنوات \\
\hline $1 \ldots$ & $Y V, 01$ & $1 \ldots$ & $\cdot, 9 \wedge \varepsilon$ & $1 \ldots$ & $r V, q V$ & r... \\
\hline $17 \cdot, 5$ & $\varepsilon \varepsilon, 1 \mu$ & QV, YO & $\cdot, 901$ & $17 \varepsilon, V \leqslant$ & $\sum 7,1$ & $r \ldots l$ \\
\hline ITV,ro & ro, s & $97,0 \leqslant$ &., 90. & $1 \Pi, \wedge T$ & $r q, 9$ & $r \ldots r$ \\
\hline $11 \varepsilon, \wedge 1$ & $T, 7$ & 99,11 & $\cdot, 9 \vee 7$ & 110,79 & $r, \varepsilon$ & $r \ldots r$ \\
\hline 171 & $\leqslant \leqslant, \Gamma$ & $9 \wedge, 91$ & $\cdot, 9 \vee \varepsilon$ & $17 r, 07$ & $\leqslant 0,0$ & $r \ldots \varepsilon$ \\
\hline $11 \cdot, 0 Y$ & $r \cdot, \leqslant 1$ & $9 \wedge, r V$ & $\cdot, 97 V$ & $11 Y, \leqslant 7$ & $M, 0$ & $r \ldots o$ \\
\hline $1 \% \cdot, \cdot 1$ & ro, VV & $I \cdot r, r$ & $1, \cdots \varepsilon$ & $I T V, \leqslant Y$ & 10,70 & $r \ldots r$ \\
\hline $1 \cdots, \varepsilon$ & $T V, T Y$ & $1 . r, r_{0}$ & $1, \cdot 1 \mathrm{~V}$ & $9 V, 1 \varepsilon$ & $r V, r$ & $r \ldots V$ \\
\hline$V \varepsilon, 1$. & $r \cdot, \xi$ & $1 \cdot V, A Y$ & 1,91 & $T \Lambda, V Y$ & $19, Y Y$ & $r \ldots \lambda$ \\
\hline $1 \leqslant Y$ & $r q, 1$ & $1 \cdot 1, \leqslant Y$ & $\cdot, 991$ & $1 \leqslant 1, V Y$ & $r 9,70$ & $r \ldots q$ \\
\hline ITr,AY & $r q, \wedge r$ & $1.7,1$ & $1, \cdot \leq \varepsilon$ & $117, .0$ & ro,r & $r .1$. \\
\hline 77,7 & $1 \wedge, \mu Y$ & $1.7, Y$ & $1,1 \leq 0$ & $7 r, 71$ & IV,Or & $r .11$ \\
\hline$V Y, T \varepsilon$ & 19,91 & $1 \leqslant \Lambda, \leqslant V$ & $1,\{71$ & TT,T & $I V, V I$ & $r .1 T$ \\
\hline 79,11 & $19, \cdot r$ & $I T V, T \varepsilon$ & $1, Y 07$ & $0 \leqslant, 19$ & $10, Y$ & $r .1 T$ \\
\hline$V \wedge, Y \varepsilon$ & YI,Or & $1 \Gamma \varepsilon, \cdot \varepsilon$ & 1,419 & $O \wedge, \mu$ & $17, r Y$ & T.1s \\
\hline $1 ., 0 \mathrm{~V}$ & $r Y, Y$ & $1 \leqslant r, 79$ & $1, \leqslant 1 \leqslant$ & 07,5 & $10, \mathrm{~V}$ & $r .10$ \\
\hline $1 \cdot V, 7$. & $r q, 7)$ & $11 \cdot, 71$ & 1,199 & $1 \cdot r, 71$ & $r \wedge, V r$ & المتوسط \\
\hline
\end{tabular}

المصدر:_وزارة الزر اعة واستصلاح الأراضي، قطاع الثئون الإقتصادية، الإدارة المركزية للإقتصساد الزر اعي، نشرة الإحصاءات الزر اعية، أعداد مختلفة.

Fayoum J. Agric. Res. \& Dev., Vol. 33, No.1, January, 2019 
$1 \leqslant V$

جدول رقم ( ) ) معادلات الاتجاه الزمني العام لتطور المسـاحة المزروعة والإنتاجية الفدانية والإنتاج الكلي

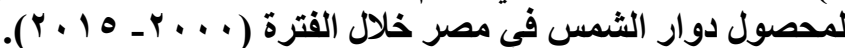

\begin{tabular}{|c|c|c|c|c|c|}
\hline |السنوى التغير \% & $\mathbf{F}$ & $\mathbf{R}^{2}$ & معادلة الاتجاه الزمني العام & المعادثة & المتغير \\
\hline $0, Y_{1}$ & $* 10, v r$ &., or & ص^ هـ= ס ץ, & 1 & المساحة المزروعة \\
\hline$r, 77$ & $* r y, y$ & $\cdot, 70$ & ص ص * & $r$ & الإنتاجية الفدانية \\
\hline$r, 9 \Lambda_{-}$ & $* q, Y V$ & $\cdot, r q$ & ** ص ص & $r$ & الإنتاج الكلي \\
\hline
\end{tabular}

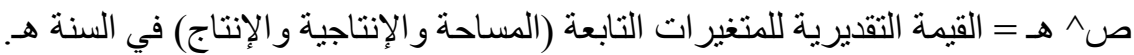

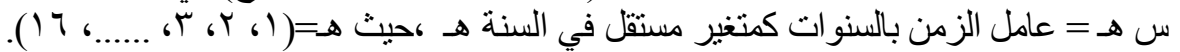

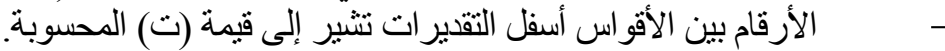

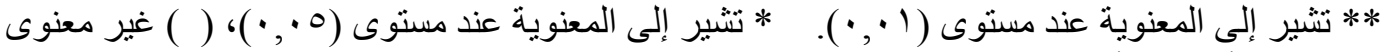

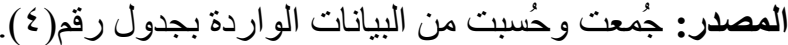

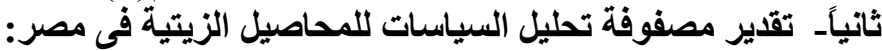

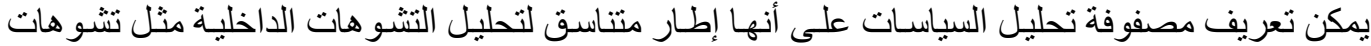

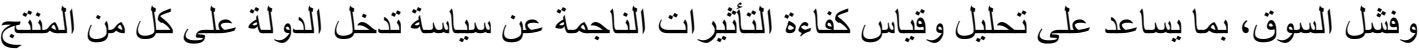

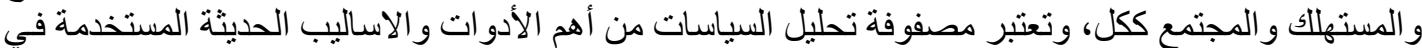

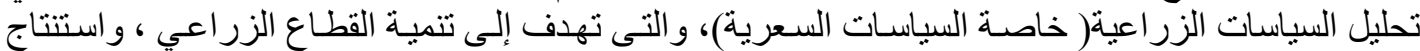

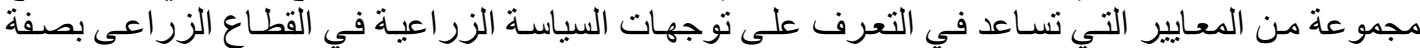

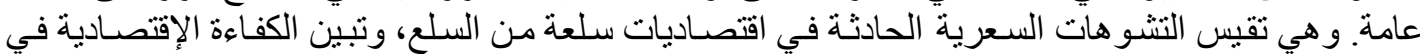

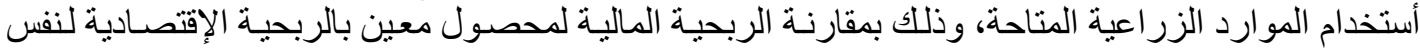

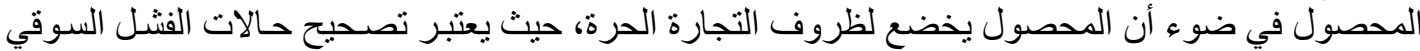

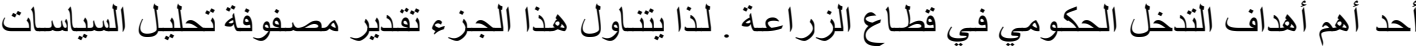

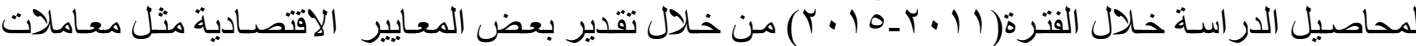

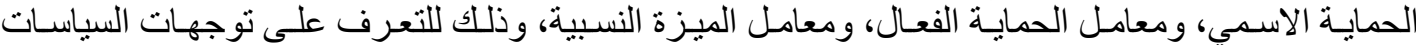

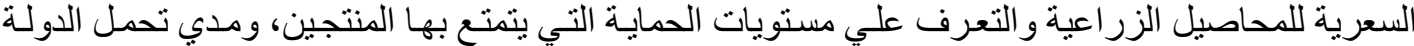

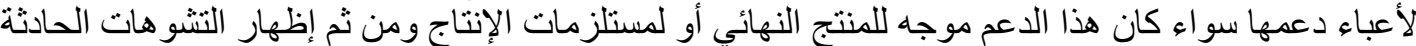

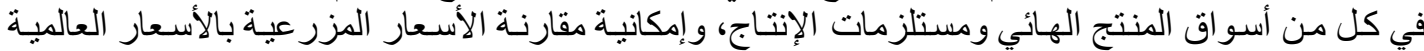

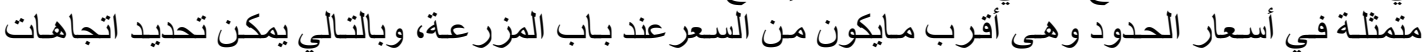

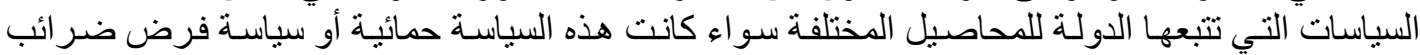

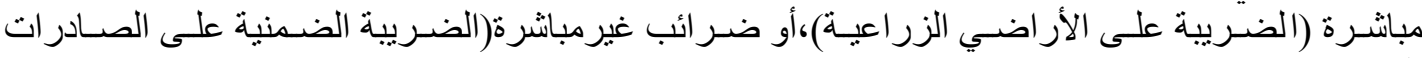

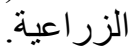

ـ معامل الحماية الأسمى للمنتجات(NPCO)=

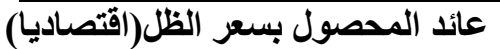

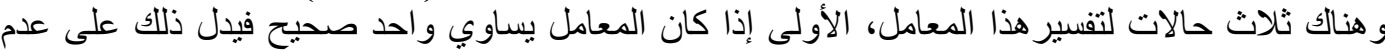

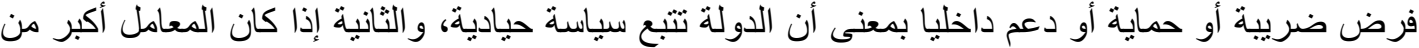

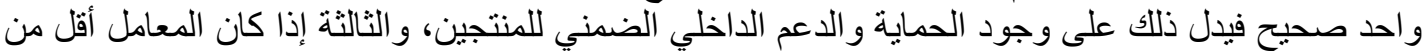

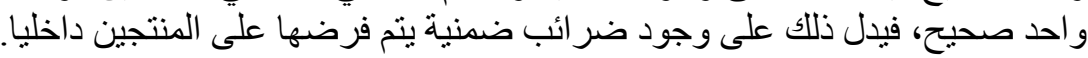

ـ معامل الحماية الأسمى لمستلزمات الإنتاج(NPCI)= قيمة مستلزمات الإتتاج بسعر السوق(ماليا)

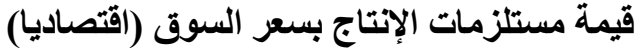

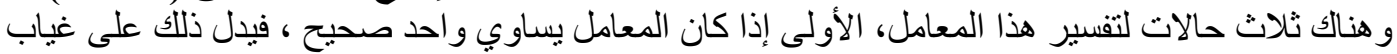

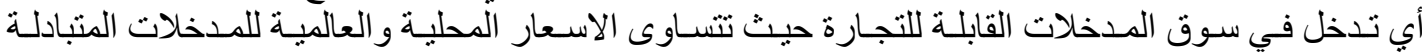

Fayoum J. Agric. Res. \& Dev., Vol. 33, No.1, January, 2019 
$1 \leqslant 1$

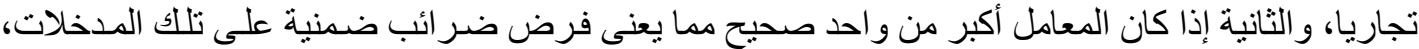

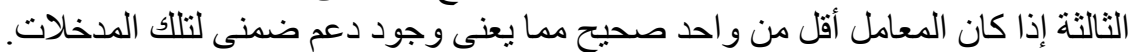

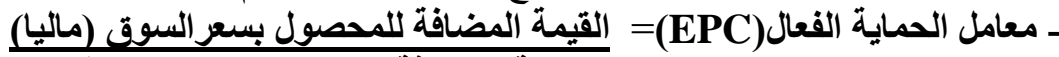

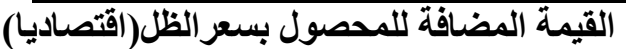

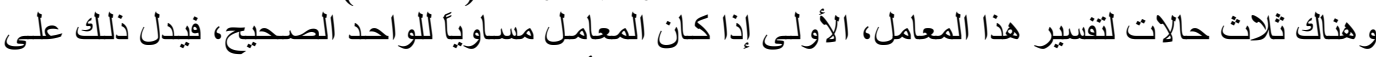

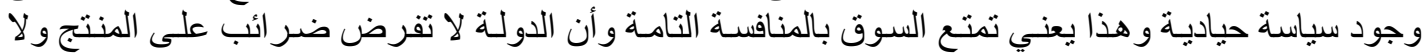

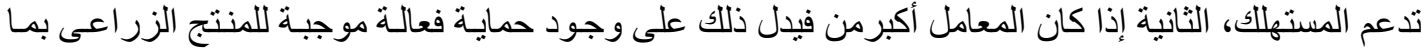

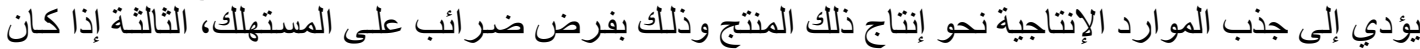

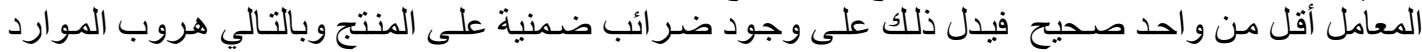

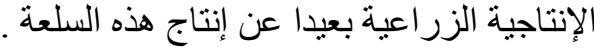

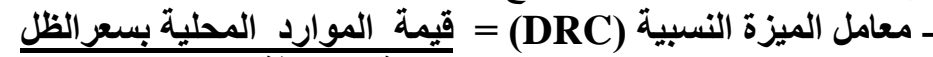

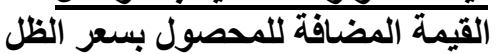

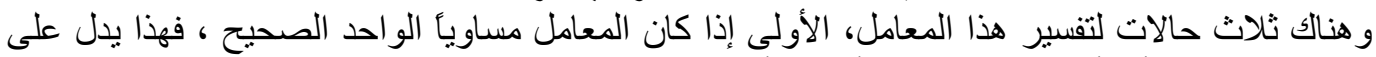

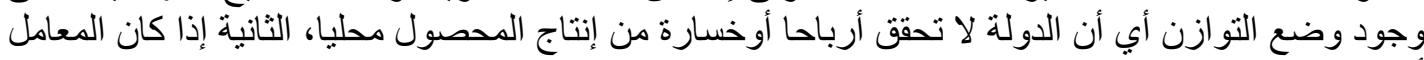

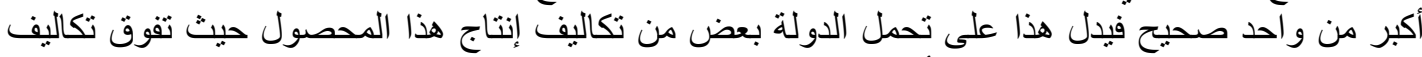

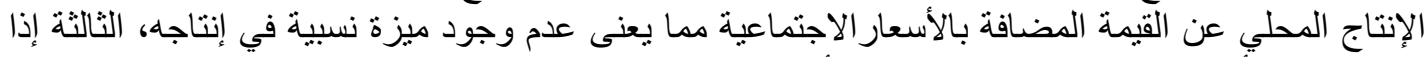

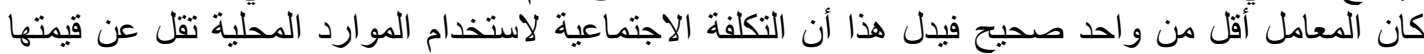

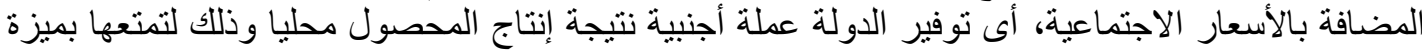

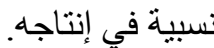
وتجدر الاشارة بانه للوصول إلى التكلفة الإنتاجية لإنتاج للمحاصيل الزيتية موضع الدر اسة مقيمة بأسعار

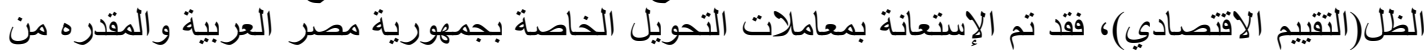

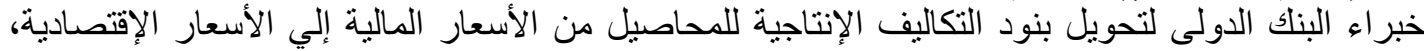

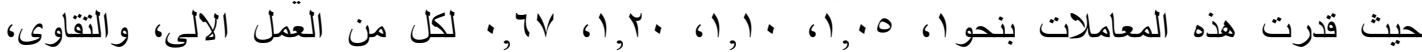

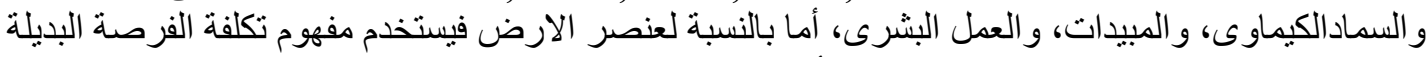

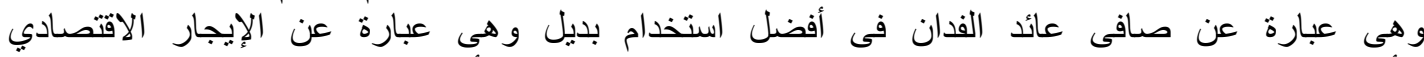

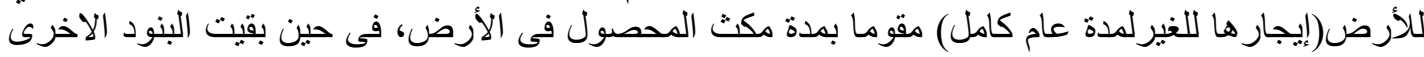

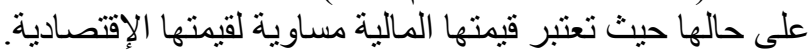

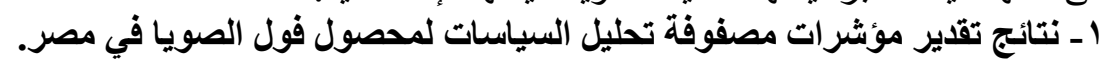

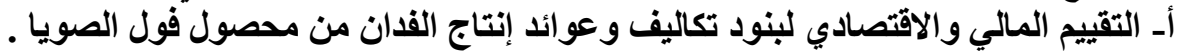

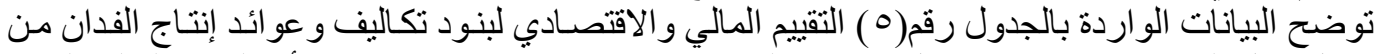

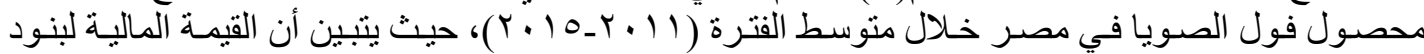

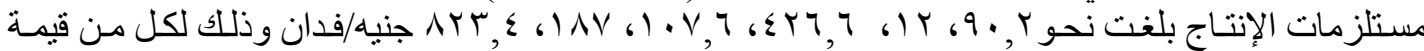

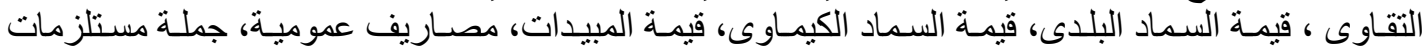

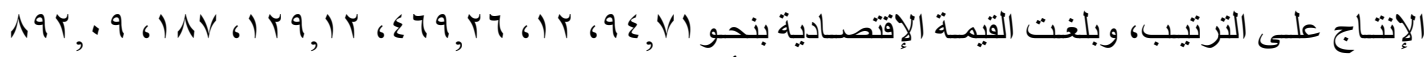

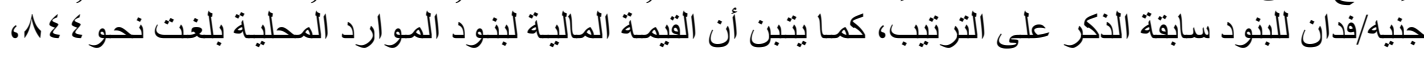

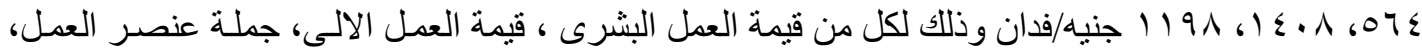

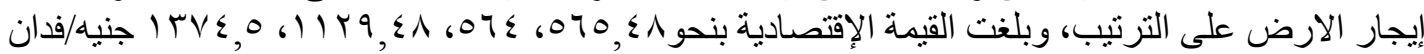

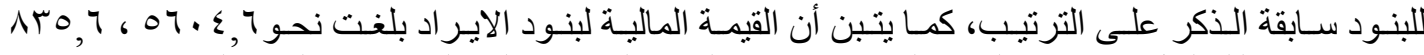

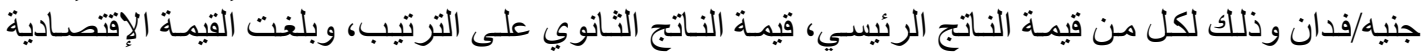

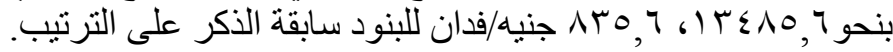

Fayoum J. Agric. Res. \& Dev., Vol. 33, No.1, January, 2019 
$1 \leq 9$

جدول رقم (ه) التقييم المالي والاقتصادي لبنود تكاليف وعوائد إنتاج الفدان من محصول فول الصويا في (القيمة بالجنيه).

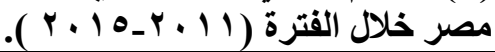

\begin{tabular}{|c|c|c|}
\hline التقييم الاقتصادي & التقييم المالي & البنود \\
\hline - & - & أو لا: مستلزمات الإنتاج \\
\hline $9 \leqslant, 71$ & $9 \cdot, r$ & قيمة التقاوي \\
\hline IT & Tr & قيمة السماد البلدي \\
\hline$\sum 79, Y 7$ & $\varepsilon r\rceil, 7$ & قيمة السماد الكبماوي \\
\hline $1 Y 9,1 \%$ & $1 \cdot v, 7$ & قيمة المبيدات \\
\hline liv & IAV & مصاريف عمومية (اخرى) \\
\hline $194, \cdot 9$ & $A T r, \varepsilon$ & جملة مستلزمات الإنتاج \\
\hline - & - & ثنانيا الموارد المحلية \\
\hline $070, \leqslant 1$ & $\Lambda \leqslant \varepsilon$ & قبمة العمل البشري \\
\hline $07 \varepsilon$ & $07 \varepsilon$ & قيمة العمل الالى \\
\hline- & - & قيمة العمل الحيو اني \\
\hline $11 Y 9, \leqslant 1$ & $1 \xi \cdot 1$ & جملة عنصر العمل \\
\hline$r \cdot Y 1,0 V$ & KMTI, & جملة التكاليف المتغيرة (بدون إيجار ) \\
\hline $1 T V \leqslant, 0$ & 1191 & إيجار الأرض \\
\hline$r r q 7, V$ & $r \leqslant r q, \varepsilon$ & جملة التكاليف الكلية \\
\hline - & - & ثالثا: الاير اد \\
\hline $1 \Gamma \leqslant \wedge 0,7$ & $07 .\{, 7$ & قيمة الناتج الرئيسي "بالجنيه \\
\hline$\Lambda \mu O, T$ & $\Lambda \mu_{0}, T$ & قيمة الناتج الثانوي "بالجنيه" \\
\hline $1 \leqslant r r \mid, r$ & $T \varepsilon \varepsilon \cdot, Y$ & جملة الإير اد \\
\hline $1.940,1 \%$ & $r \cdot 1 \cdot \lambda$ & صافى العائد \\
\hline
\end{tabular}

المصدر: بيانات التقييم المالي، جمعت وحسبت من بيانات وزارة الزر اعة واستصلاح الأراضي، قطاع الثشئون

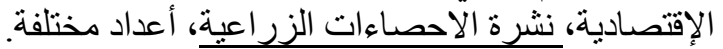

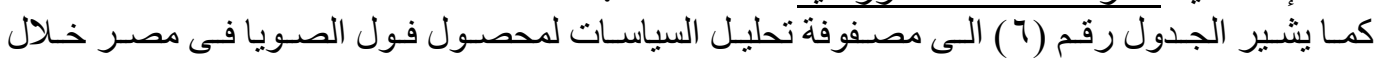

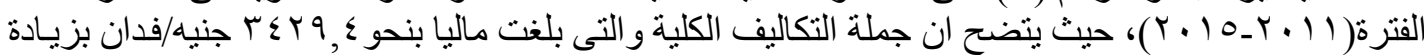
قدر هاس

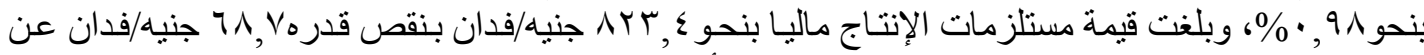

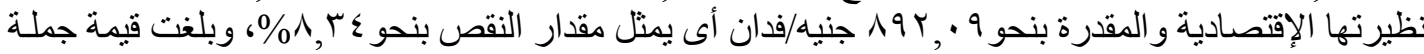

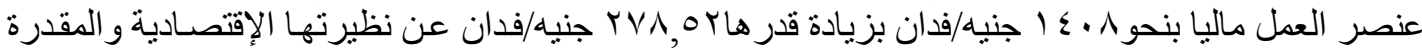

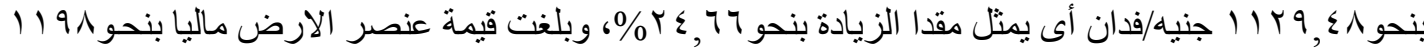

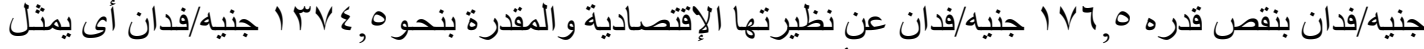

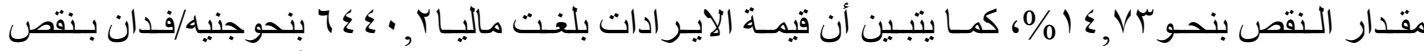

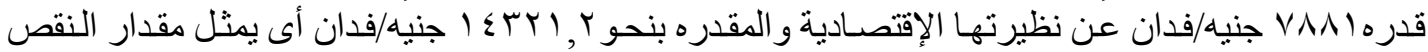

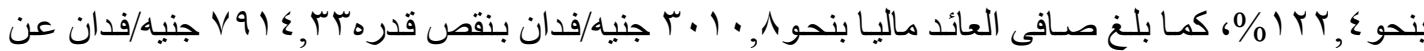

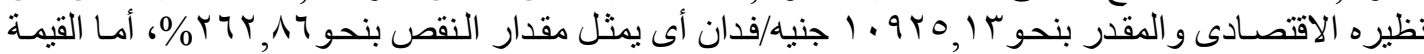

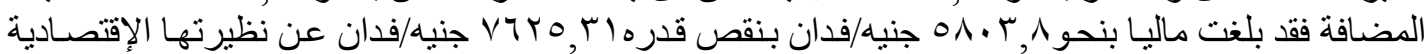

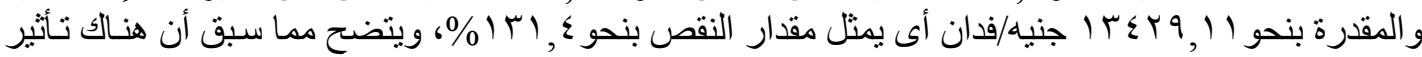

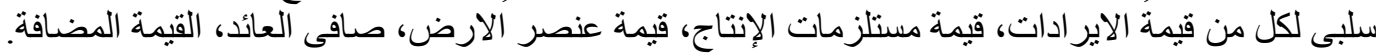

Fayoum J. Agric. Res. \& Dev., Vol. 33, No.1, January, 2019 
10.

جدول رقم (7) تقدير مصفوفة تحليل السياسـات لمحصول فول الصـويا في مصرخلال القترة (11 ـ بـ

(القيمة بالجنيه)

. ( $r .10$

\begin{tabular}{|c|c|c|c|c|c|c|c|}
\hline \multirow[b]{2}{*}{ القيمة المضافة } & \multirow[b]{2}{*}{ صافي العائد } & \multirow[b]{2}{*}{ |تكاليف كلية } & \multicolumn{2}{|c|}{ الموارد المحلية } & \multirow{2}{*}{ 柔率 } & \multirow{2}{*}{ الإيرادات } & \multirow[b]{2}{*}{ البيان } \\
\hline & & & الأرض & عنصر العمل & & & \\
\hline $0 \Lambda \cdot r, \Lambda$ & $r \cdot 1 \cdot, \wedge$ & $r \leqslant r q, \varepsilon$ & 1191 & $1 \varepsilon \cdot 1$ & Arr, & $T \leq \varepsilon, r$ & 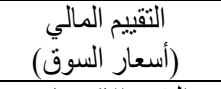 \\
\hline $\mid r \leqslant r q, 11$ & $1.9 r 0,15$ & rrq,$v$ & $1 T V \leqslant, 0$ & $11 r q, \varepsilon \wedge$ & $194, \cdot 9$ & $1 \leqslant \pi r \mid, r$ & 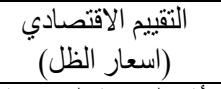 \\
\hline VTro,ri. & vq $\{, r r-$ & $\mu$ & $1 \times 4,0_{-}$ & rVA,Or & 71,79 & VAMI- & أثر السياسة الزر اعية \\
\hline
\end{tabular}

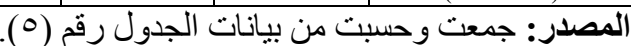

بـ تقدير مؤشرات مصفوفة تحليل السياسات لمحصول فول فول الصويا.

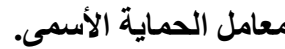

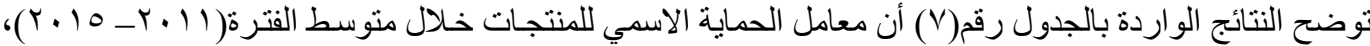

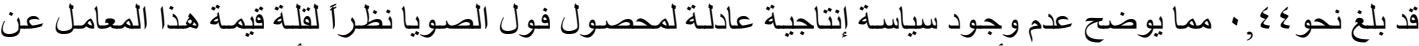

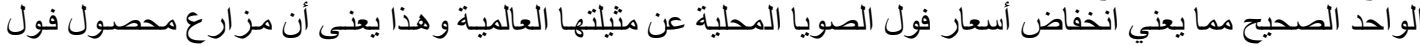

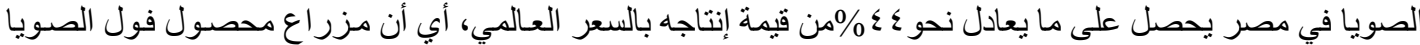

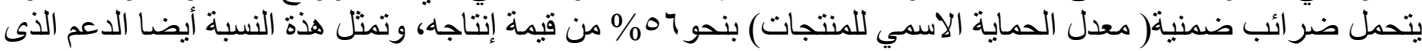

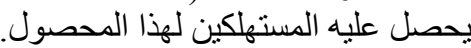

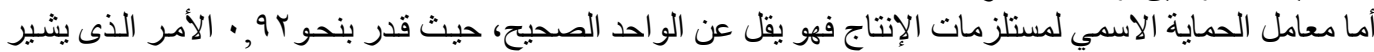

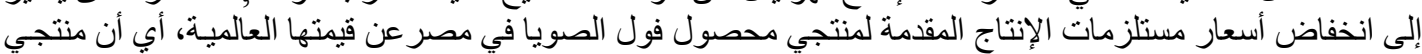

المحصول في مصر يتلقون دعما إمنيا.

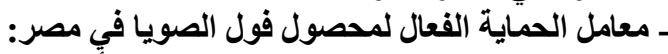

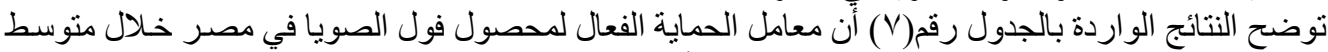

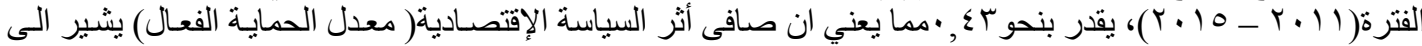

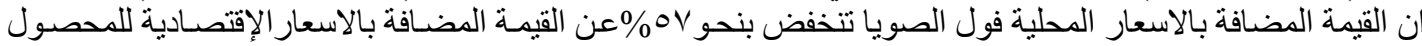

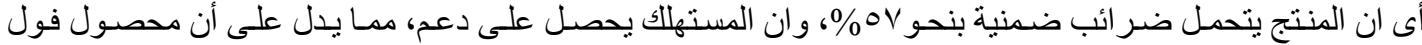
الصويا لا بتمتع بحماية حكومية.

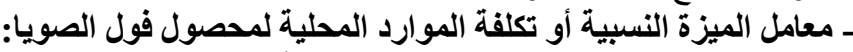

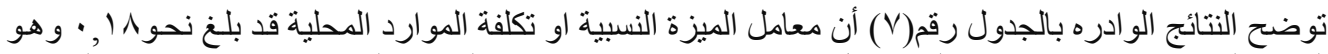

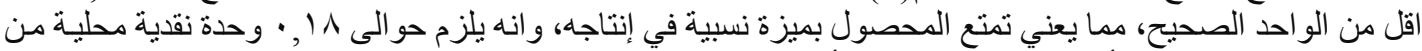

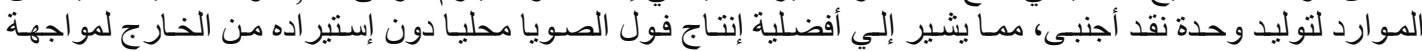

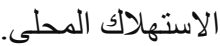
جدول رقم(V) نتائج تقدير مؤشرات مصفوفة تحليل السياسة لمحصول فول الصويا في مصر خلال الفترة (11 + ؟ـ . ( $r .10$

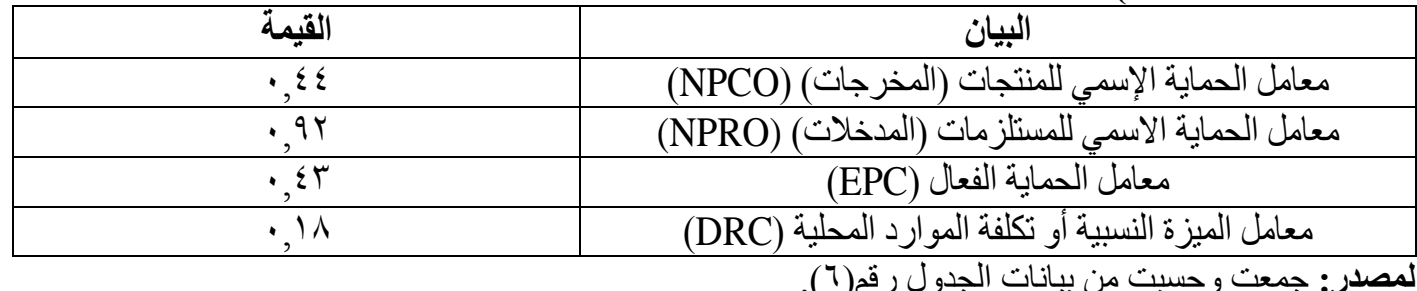

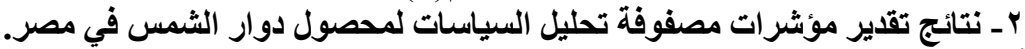

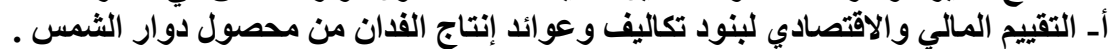

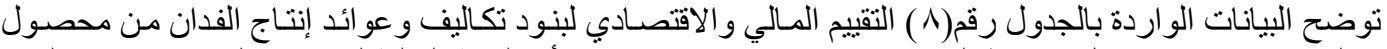

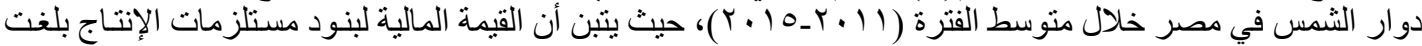

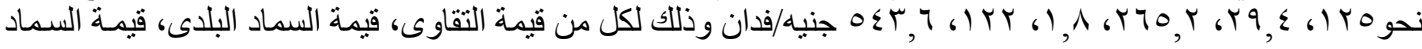

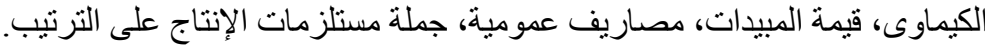

Fayoum J. Agric. Res. \& Dev., Vol. 33, No.1, January, 2019 
جدول رقم (^) التقييم المالي والاقتصادي لبنود تكاليف وعو ائد إنتاج الفدان من محصول دوار الثمس في مصر خلال (القيمة بالجنيه) (الفيان)

\begin{tabular}{|c|c|c|}
\hline التقييم الاقتصادي & التقيميم المالي & البنود \\
\hline- & - & أو لا: مستلزمات الإنتاج \\
\hline $1 \pi 1, r_{0}$ & 1ro & قيمة التقاوي \\
\hline rq, $\varepsilon$ & $r q, \xi$ & قيمة السماد البلدي \\
\hline Yq1,Vr & rTo,r & قيمة السماد الكيماوي \\
\hline$r, 17$ & 1,1 & قيمة المبيدات \\
\hline TYK & TYK & مصاريف عمومية (اخرى) \\
\hline OV7,Or & $0 \leqslant Y, 1$ & جملة مستلزمات الإنتاج \\
\hline- & - & ثانيا المو ارد المحلية \\
\hline$\varepsilon r \cdot, \cdot 9$ & $\pi T V$ & قيمة العمل البشري \\
\hline$r r v, \Lambda$ & $r r v, \Lambda$ & قيمة العمل الالى \\
\hline- & - & قيمة العمل الحيو انى \\
\hline VOV,Aq & $97 \leqslant, \wedge$ & جملة عنصر العمل \\
\hline$T M Y \leqslant, \varepsilon T$ & $10 \cdot 1,5$ & جملة التكاليف المتغيرة (بدون إيجار) \\
\hline$|Y| \leqslant, V$ & $1 \cdot V Y, \xi$ & إيجار الأرض \\
\hline ro $9,1 r$ & $r \circ \Lambda \cdot, \Lambda$ & جملة التكاليف الكلية \\
\hline- & - & ت ثالثا: الاير اد \\
\hline ANT7, Y & $r 9.7,1$ & قيمة الناتج الرئيسي "بالجنيه \\
\hline$r \cdot \Lambda, T$ & $r \cdot \Lambda, T$ & قيمة الناتج الثانوي "بالجنيه" \\
\hline $9 \cdot \vee \leqslant, \wedge$ & $\leqslant 110, \leqslant$ & جملة الإير اد \\
\hline 10Y0,Y & $10 r \leqslant, 7$ & صافي العائد \\
\hline
\end{tabular}

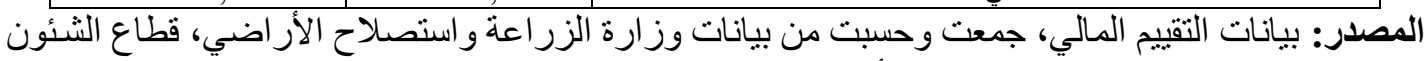

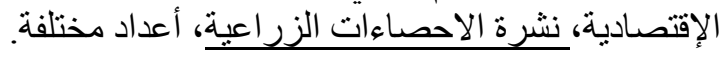

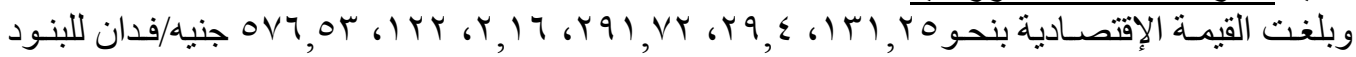

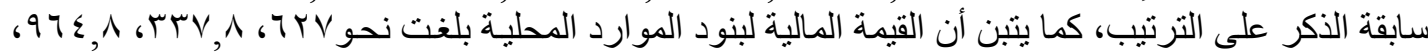

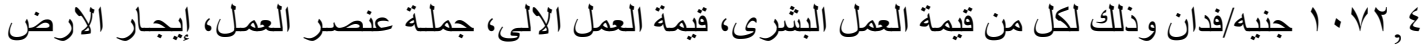

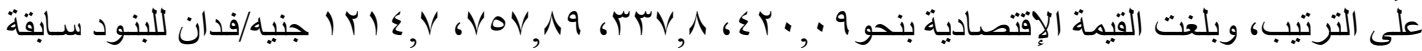

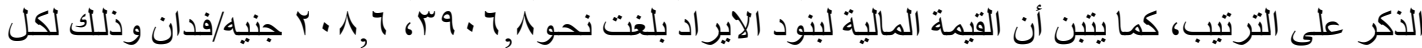

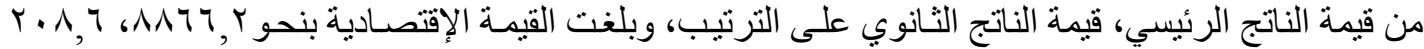

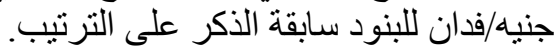

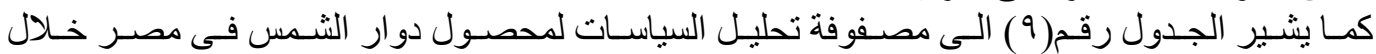

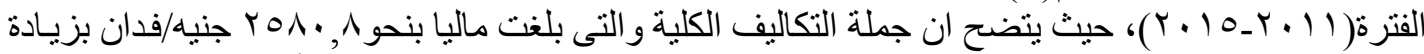

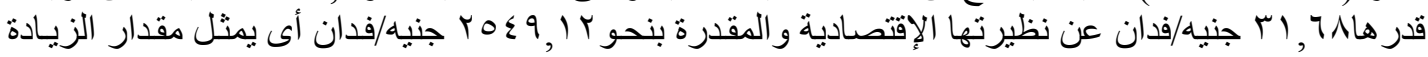

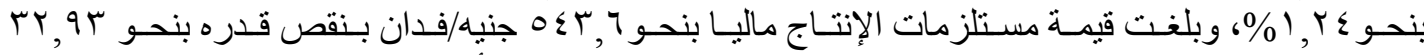

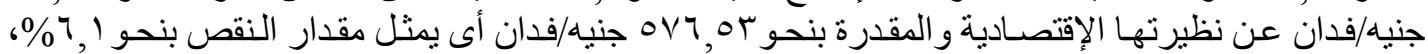

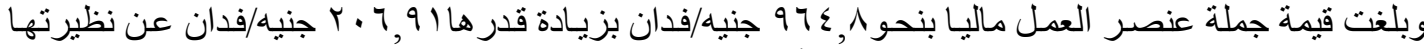

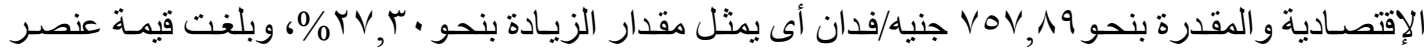

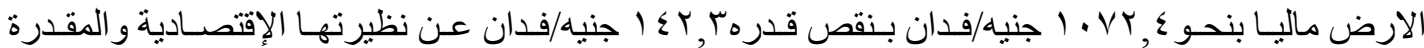

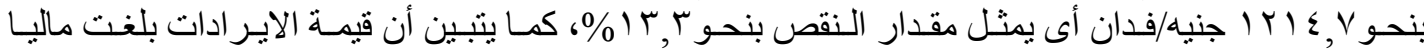

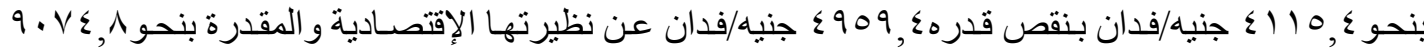

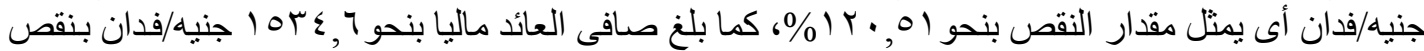

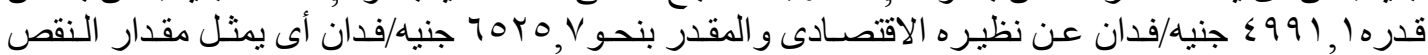

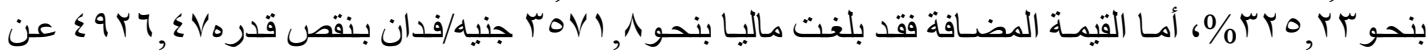

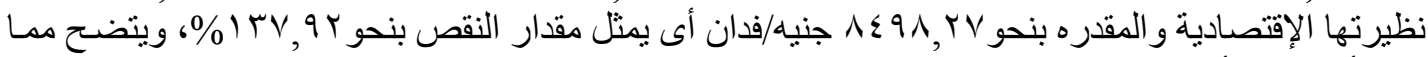

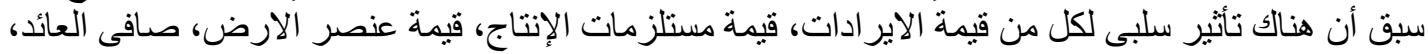

Fayoum J. Agric. Res. \& Dev., Vol. 33, No.1, January, 2019 
10r

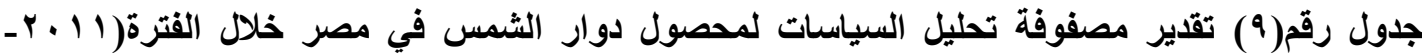
(القيمة بالجنيهة)

$(r \cdot 10$

\begin{tabular}{|c|c|c|c|c|c|c|c|}
\hline \multirow{2}{*}{ المضافة } & \multirow{2}{*}{ صافي } & \multirow{2}{*}{ كلية } & \multicolumn{2}{|c|}{ الموارد المحلية } & \multirow{2}{*}{ مستلزمات } & \multirow[t]{2}{*}{ الإيرادات } & \multirow{2}{*}{ البيان } \\
\hline & & & الأرض & عر العمل & & & \\
\hline rovi,1 & $r \leqslant, 7$ & rod., A & $1 \cdot V T, \varepsilon$ & $97 \leqslant, 1$ & $0 \leqslant r, 7$ & $\leqslant 110, \leqslant$ & بعار السوق) \\
\hline$\Lambda \leq 9 \Lambda, Y V$ & 70Y0, & $r 0 \leqslant 9,1 Y$ & $|r| \leqslant, V$ & $V \circ V, \wedge 9$ & oVy,or & $9 \cdot V \leqslant, 1$ & ب (اسعار الظل) \\
\hline$q Y \uparrow, \leqslant V_{-}$ & $\leqslant 991,1$. & $r$ & $1 \leqslant r$ & $r \cdot 7,91$ & rr,qr_ & $\leqslant 909, \varepsilon-$ & لزر اعبة (التحويلات) \\
\hline
\end{tabular}

المصدر: جمعت وحسبت من بيانات الجدول رقم (^).

ب- تقدير مؤشرات مصفوفة تحليل السياسات لمحصول دوار الثمس.

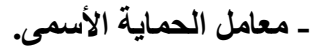

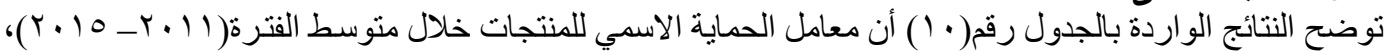

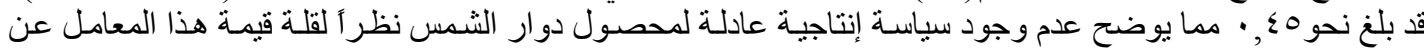

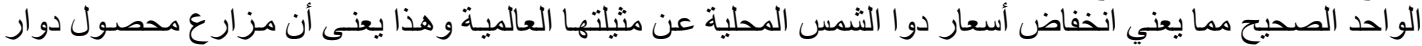

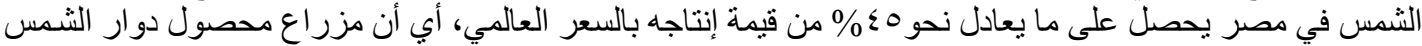

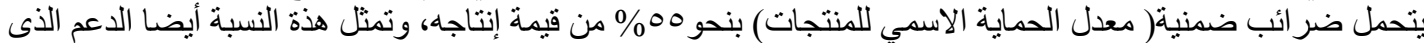
يحصل عليه المستهلكين لهذا المحصول.

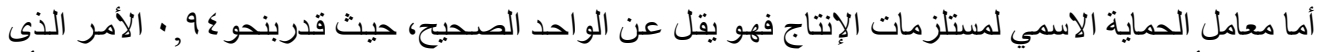

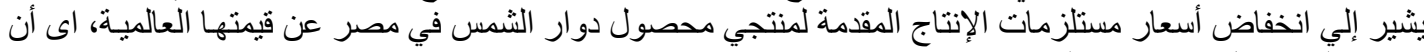
منتجي المحصول في مصر يتلقون دعما ضمنيل الفماضيا.

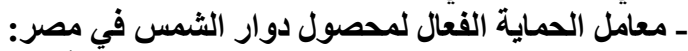

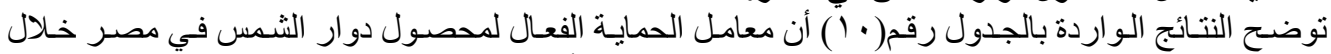

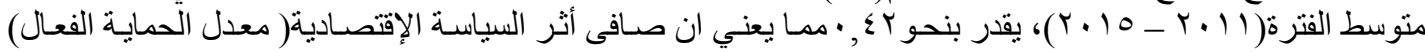

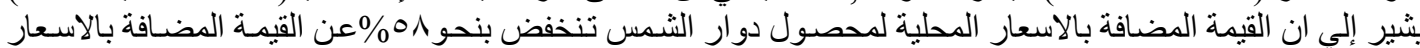

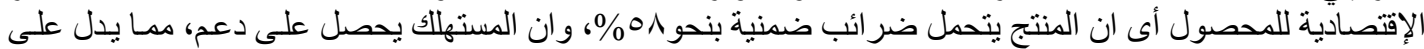

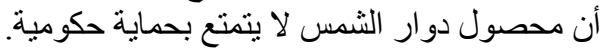

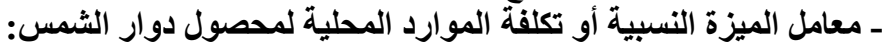

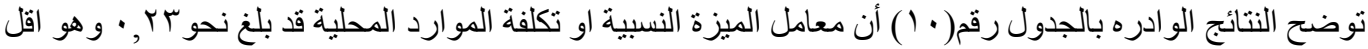

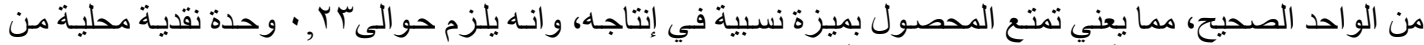

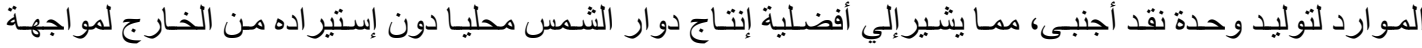

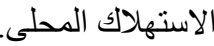
جدول رقم( • 1) نتائج تقدير مؤشرات مصفوفة تحليل السياسة لمحصول دوار الشمس في مصر خلال القترة (1 1 ـ ب ـ . .$(Y+10$

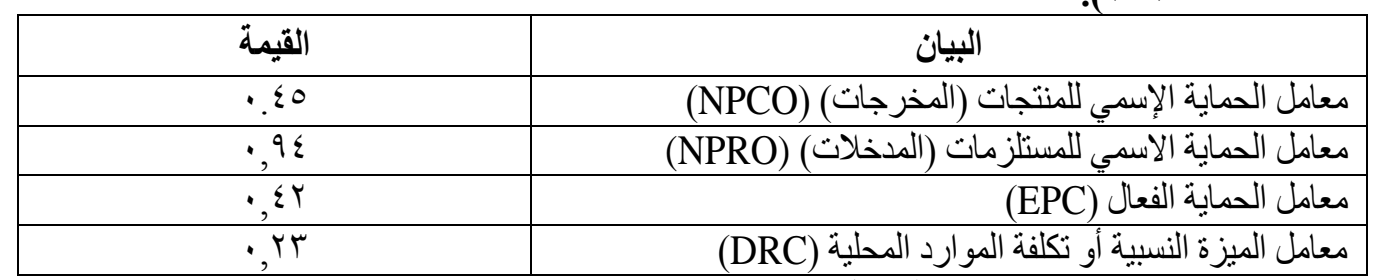

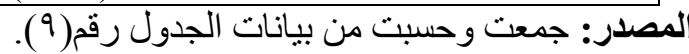

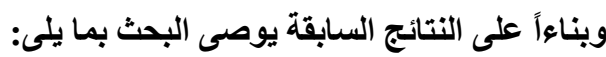

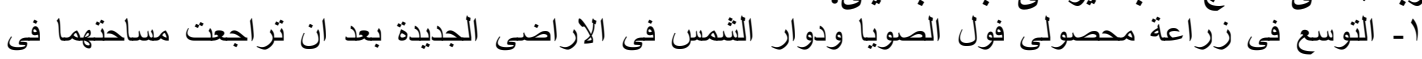
الار اضنى القديمة.

rـ التوسع فى نظام الزر اعة التعاقدية للمحاصيل الزيتية نظر آ لانها محاصيل تصنعية.

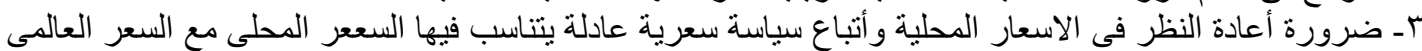

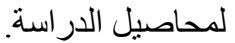
ع - ضرورة الاهنمام بالسياسات الزر الزية الخاصة بدعم المنتج.

Fayoum J. Agric. Res. \& Dev., Vol. 33, No.1, January, 2019 


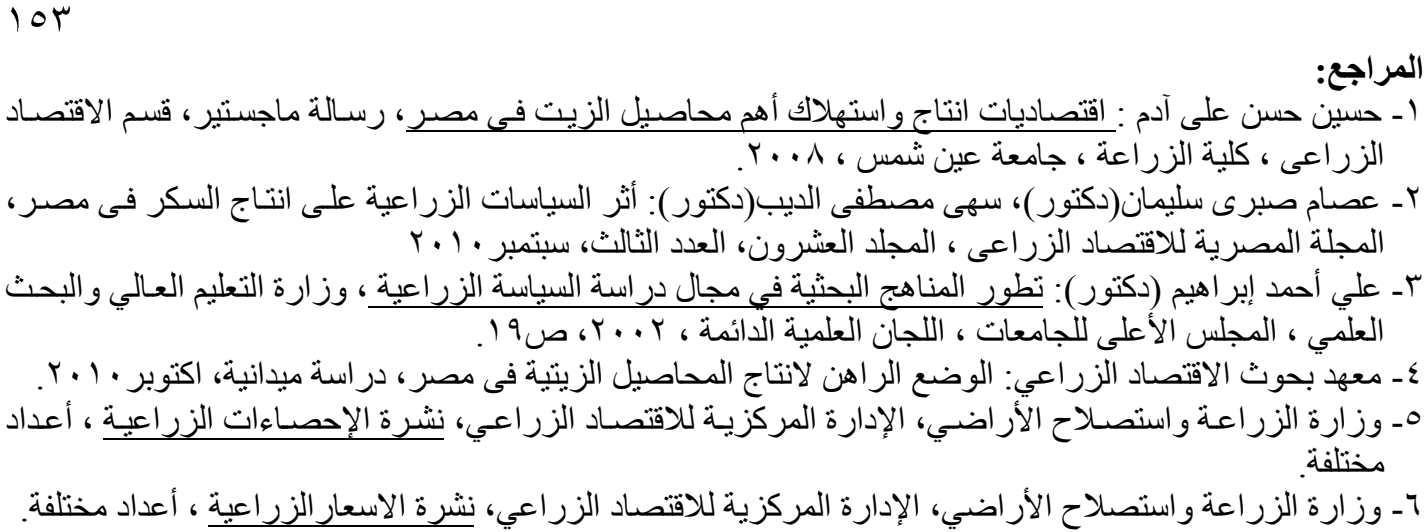

\section{ANALYTICAL STUDY FOR THE IMPACT AGRICULTURAL AND PRICE POLICIES ON PRODUCTION AND CONSUMPTION OF SOME CROPS OILSEED IN EGYPT}

*Enas El Sayed Sadek, *Ayman Abd Al kawy Shelaby **Gamal El Sayed Mohamed **Ahmed, Hussein Qurany Sayed Said.

*Faculty Of Agriculture, Fayoum University. **Agricultural Economics Research Institute, Agricultural Research Center .

\section{ABSTRACT}

Oil crops are important crops in Egypt because they are the main source of vegetable oil production in the food scale. (60\%) of the animal wealth in Egypt, as well as the fact that they enter many food industries, and the importance of that wealth, it is not possible to verify the size of the resistance in real time oil.

The results of the research showed that the average size of the farm in Fawaya was about 21.55 thousand feddans during the period 2000-2015. The average feddan reached about 1,341 tons / fed. (2000-2015), the average feddan yield is about 1,089 tons/fed, in the period of California For the yield of about 29.61 thousand tons. In estimating the general time trend equation for the evolution of the study variables of the crop, At a statistically significant annual rate, while productivity increases at a statistically significant annual rate.

Based on the findings of the Fund's Profile Analysis Matrix (2000-2015), the protection coefficient for fish may be below the reasonable price of 0.44 , which means that domestic soybean prices are lower than their global counterparts, ie the soybean crop bears implicit taxes Approximately $56 \%$ of the value of its production, such a presentation of the United States which is close to the minimum, $0.92 \%$, indicating that the producers of the crop in Egypt are implicitly subsidized, and the effective protection factor line below 0.43 which means that the product bears implicit taxes 57 And that the consumer receives support, as indicated by the coefficient of comparative advantage, that the final Egypt is relative $\mathrm{C}$ crop without imported from abroad, according to the camp .18 ratio, which is less than the correct one.

As shown by the results of the military file analysis matrix in Egypt during the period 20002015 , the nominal protection coefficient for the related products is 0.45 , which means that the prices of the local sunflower are lower than their global counterparts. Of the value of its production, such as that of Nindhoville, led to be produced everywhere, $0.94 \%$, which indicates that the producers of the crop in Egypt are implicitly subsidized, as the coefficient of effective protection coefficient is $0.42 \%$ about $58 \%$, and the consumer It also receives support, as the comparative advantage indicates that Egypt has a comparative advantage in producing the crop without importing it from outside to face it Where its domestic consumption amounted to about 0.23 , less than the right one.

Key words: Nominal protection coefficients-effective protection coefficient- coefficient of comparative advantage.

Fayoum J. Agric. Res. \& Dev., Vol. 33, No.1, January, 2019 